\title{
Pediatric Infectious Disease Terminology
}

National Cancer Institute

\section{Source}

National Cancer Institute. Pediatric Infectious Disease Terminology. NCI Thesaurus.

Code C128453.

Terminology developed by a collaborative effort of the National Cancer Institute's Enterprise Vocabulary Services, the National Institute of Child Health and Human Development, and an international group of clinicians and informaticists to support the reporting of events in the pediatric population with issues pertaining to infectious diseases. 